\title{
Use of next-generation amplicon sequencing to study Blastocystis genetic diversity in a rural human population from Mexico
}

Liliana Rojas-Velázquez ${ }^{1,3 \dagger}$, Jenny G. Maloney ${ }^{2 \dagger}$, Aleksey Molokin² ${ }^{2}$ Patricia Morán ${ }^{1}$, Angélica Serrano-Vázquez ${ }^{1}$, Enrique González ${ }^{1}$, Horacio Pérez-Juárez ${ }^{1,3}$, Cecilia Ximénez ${ }^{1 *}$ and Monica Santin ${ }^{2 *}$

\begin{abstract}
Background: The intestinal parasite Blastocystis is found in humans and animals around the world. It is spread through the consumption of contaminated food and water and has been associated with a variety of intestinal symptoms. Blastocystis is one of the most common intestinal parasites in humans, yet its prevalence and distribution in humans in North America is not well characterized.

Methods: Next-generation amplicon sequencing of a region of the Blastocystis SSU rRNA gene was applied to DNA extracted from fecal specimens obtained from 182 inhabitants of a rural population in Mexico to characterize Blastocystis prevalence, subtype distribution, and intra-host subtype diversity in humans.

Results: Of the 182 samples tested in this study, 68.1\% (124) contained one or more Blastocystis subtypes. Subtype 3 was the most common subtype observed and was found in $81.5 \%$ of the positive samples. Subtype 1, 16.9\% of the positive samples, and subtype $2,17.7 \%$ of the positive samples, were also found in this population. Mixed infections were observed in $13.7 \%$ of the positive samples. In this population, the odds of having Blastocystis increased in adulthood (> 15 years; OR: 1.72, $P<0.0001$ ), and the odds of having subtype 1 increased in the presence of farm animals (OR: 1.51, $P=0.03$ ). The odds of having subtype 1 , subtype 2 , or a mixed infection decreased in the presence of cement flooring (OR: $-1.61, P=0.005 ; \mathrm{OR}:-1.14, P=0.03$; OR: $-1.48, P=0.02$ ) possibly indicating socioeconomic factors are involved in the risk of acquiring one of these subtypes.

Conclusions: These data contribute to our understanding of the epidemiology of Blastocystis infection in humans and can be used to shape future studies which aim to better characterize the transmission pathways and health outcomes of Blastocystis infections.
\end{abstract}

Keywords: Blastocystis, Human, Mexico, Mixed infections, Next-generation sequencing, Risk factors, Subtypes

\footnotetext{
*Correspondence: cximenez@unam.mx; Monica.Santin-Duran@ars.usda.gov

†Liliana Rojas-Velázquez and Jenny G. Maloney contributed equally to

this work

${ }^{1}$ Unidad de Investigación en Medicina Experimental, Facultad de

Medicina, Universidad Nacional Autónoma de México (UNAM), Mexico

City, Mexico

${ }^{2}$ Environmental Microbial and Food Safety Laboratory, Agricultural

Research Service, United States Department of Agriculture, Beltsville, MD,

USA

Full list of author information is available at the end of the article
}

(c) The Author(s) 2019. This article is distributed under the terms of the Creative Commons Attribution 4.0 International License (http:/creativecommons.org/licenses/by/4.0/) which permits unrestricted use, distribution, and reproduction in any medium, provided you give appropriate credit to the original author(s) and the source, provide a link to the Creative Commons license, and indicate if changes were made. The Creative Commons Public Domain Dedication waiver (http://creativecommons.org/ publicdomain/zero/1.0/) applies to the data made available in this article, unless otherwise stated. 


\section{Background}

Blastocystis is a cosmopolitan enteric parasite found in humans and a wide range of animals across the world. Currently, Blastocystis is the most common intestinal parasite in humans in developing and developed countries [1]. However, the role of Blastocystis as a pathogen is still controversial, mainly because it is found in both patients suffering with intestinal symptomatology, such as diarrhea, flatulence, bloating or abdominal discomfort, as well as in healthy people [2]. Blastocystis has also been associated with irritable bowel syndrome and with cutaneous symptoms (urticaria) [2, 3]. Blastocystis is transmitted via the fecal-oral route either indirectly through ingestion of food or water contaminated with cysts or directly by contact with infected persons or animals [4].

A high degree of genetic diversity has been found among Blastocystis isolates based on nucleotide differences in the small subunit $(S S U)$ of the ribosomal RNA (rRNA) gene. So far, at least 26 subtypes (STs) have been proposed [5-7]. Ten subtypes, ST1-ST9 and ST12, have been reported in humans, and of these subtypes all but ST9 are also found in other mammalian and avian hosts indicating the potential for zoonotic transmission $[8,9]$. Of the ten subtypes reported in humans, ST1 to ST4 are most commonly found in humans worldwide [10]. Blastocystis in humans in North America is not well characterized at the molecular level. In fact, in the USA, only one molecular survey of Blastocystis has been performed in humans and found ST1, ST2 and ST3 were all present in humans from Colorado [11]. In Mexico, most molecular studies have been conducted in patient populations to understand the association between Blastocystis and irritable bowel syndrome and have reported ST1, ST2, ST3 and ST7 in humans $[12,13]$.

Molecular characterization to identify subtypes present in samples is critical to unravel Blastocystis epidemiology and to characterize subtype level differences in host specificity, transmission, public health significance, and pathogenicity. Mixed subtype infections are often overlooked in molecular studies of Blastocystis, and a better characterization of these infections is needed to fully understand the epidemiology of Blastocystis. Recently, it was demonstrated that next-generation amplicon sequencing is a powerful tool to investigate mixed infections and detect low abundance subtypes of Blastocystis [14]. The aim of the present study was to investigate Blastocystis in a rural population from Mexico using next-generation amplicon sequencing to better characterize Blastocystis prevalence, subtype distribution, and intra-host subtype diversity in humans and to evaluate potential association of socioeconomic factors with Blastocystis infection in this population.

\section{Methods}

\section{Study population}

One hundred and eighty-two volunteers living in the community of Xoxocotla, State of Morelos (Mexico) participated in the study conducted between May and November 2014 that included 86 males, 96 females, 66 children ( $\leq 15$ years-old), and 116 adults ( $>15$ years-old) with age ranging from 2 to 51 years (median age of 23 years). Each participant provided three fecal samples that were collected on three consecutive days. The samples were maintained at $4{ }^{\circ} \mathrm{C}$ and transported to the laboratory in Mexico City. The sample size was calculated considering the total number of inhabitants in Xoxotla $(21,074)$. The minimum required sample size was calculated to be 96 individuals based on an expected frequency of intestinal parasitic infection of $50 \%$, the worst acceptable level was $10 \%$, the confidence level was $95 \%$, and the results were considered statistically significant when $P<0.05$.

\section{DNA extraction}

A similar volume of the three samples provided by each participant was combined and mixed thoroughly. Then, an aliquot of $250 \mathrm{mg}$ from the mixture was used to extract genomic DNA using the QIAamp DNA Stool Mini Kit (Qiagen, Hilden, Germany) per manufacturer's instructions. DNA was stored at $-20{ }^{\circ} \mathrm{C}$ until further molecular analysis.

\section{Molecular detection, NGS amplicon library preparation and bioinformatic analysis}

Next-generation amplicon sequencing libraries were prepared as previously described [6]. Briefly, all samples were screened by PCR using primers ILMN Blast505_532F and ILMN_Blast998_1017R. These primers amplify a region of the $S S U$ rRNA gene and are identical to Blast505_532F/Blast998_1017R [15], with the exception of containing the Illumina overhang adapter sequences on the $5^{\prime}$-end. Final libraries were quantified using the Quant-iT dsDNA Broad-Range Assay Kit (Thermo Fisher Scientific, Waltham, MA, USA) on a SpectraMax iD5 (Molecular devices, San Jose, CA, USA) prior to normalization. A final pooled library concentration of 8 pM with $20 \%$ PhiX control was sequenced using Illumina MiSeq 600 cycle v3 chemistry (Illumina, San Diego, CA, USA). Paired end reads were processed and analyzed with an in-house pipeline that uses the BBTools package v38.22 [16], VSEARCH v2.8.0 [17], and BLAST+ 2.7.1. After removing singletons, clustering and the assignment of centroid sequences to operational taxonomic units (OTU) was performed within each sample at a $98 \%$ identity threshold. Only those OTUs with a minimum of 100 sequences were retained. All raw fastq files 
were deposited to the NCBI sequence read archive under the accession number PRJNA523857. The nucleotide sequences for unique OTUs obtained in this study have been deposited in GenBank under the accession numbers MK874780-MK874822.

\section{Sociodemographic variables}

At the time of providing the samples, a questionnaire was administered to collect information on the following variables: age (child $\leq 15$ years-old or adult $>15$ years-old), gender (male or female), presence of symptoms (present or absent), type of flooring (dirt or cement), water source (city or other source), sewage disposal (in-house or other disposal), presence of animals (livestock, poultry, or companion), and presence of house pests (present or absent). The status of symptomatic was defined per the ROME III criteria commonly used by clinicians to classify gastrointestinal disorders.

\section{Data analysis}

Logistic regression analysis was used to identify factors associated with Blastocystis infection. The following demographic and socioeconomic variables were included: age (child or adult), presence of symptoms defined as answering yes to one or more Rome III criteria (asymptomatic or symptomatic), type of flooring (dirt or cement), water source (city water or other source), sewage disposal (in-house or other source), presence of domestic animals (yes or no), presence of farm animals (yes or no), presence of chickens (yes or no), and presence of house pests (yes or no). Collected sociodemographic information for this population is presented in Table 1. $P$-values $<0.05$ were considered statistically significant. Statistical analyses were performed using $\mathrm{R}$ version 3.5.1 (R Core Team, 2018).

\section{Results}

\section{Blastocystis prevalence}

Of the 182 samples screened $68.1 \%$ (124) were found to contain Blastocystis by PCR. A higher prevalence was observed in adults (81.9\%; 95/116) than in children $(43.9 \% ; 29 / 66)$, and in females $(62 \% ; 54 / 87)$ than in males (74\%; 70/95) (Table 1). A similar prevalence was found for asymptomatic $(68 \%$; $98 / 144)$ and symptomatic $(68 \%$; 26/38) participants (Table 1 and Fig. 1).

\section{Blastocystis subtypes identified using next-generation amplicon sequencing}

All 124 PCR-positive samples were sequenced using the MiSeq platform. A total of 17,514,676 read pairs were generated from the samples included in this study with an average of 141,247 reads per sample. Following trimming, pair merging, and quality filtering there were a total of 4,968,142 merged reads. After chimera filtering 4,784,056 remained. Clustering yielded 176 Blastocystis OTUs across the 124 Blastocystis-positive samples of which 43 (24.4\%) OTUs were unique (Table 2).

Three Blastocystis subtypes (ST1, ST2 and ST3) were detected in this study. Mono-subtype infections were more common than mixed infections (more than one subtype present in a single sample) representing $86.3 \%$ $(n=107)$ and $13.7 \%(n=17)$ of the positive samples, respectively (Table 3; Additional file 1: Table S1). Subtype 3 was the most frequently observed subtype in this population and was found in $81.5 \%(n=101)$ of the positive samples either as mono-infection $(n=84)$ or a mixed infection $(n=17)$ (Table 3$)$. Subtypes 1 and 2 were observed in $16.9 \%(n=21)$ and $17.7 \%(n=22)$ positive samples, respectively, as either mono-infections (9 ST1 and 14 ST2) or mixed (12 ST1 and 8 ST2). A mix of ST1 and ST3 was the most common subtype combination and was found in $52.9 \%(n=9)$ of the mixed infection samples. A mix of ST2 and ST3 was observed in 29.4\% $(n=5)$ of mixed infections, and a mix of ST1, ST2 and ST3 was observed in $17.6 \%(n=3)$ of the mixed infection samples. No ST1 and ST2 mixed infections were detected (Table 3).

\section{Intra-subtype variability}

Forty-three unique OTUs were detected among the three Blastocystis subtypes present in this study. Subtype 1 and 2 had similar intra-subtype diversity in this study with 15 unique OTUs among 21 ST1-positive samples and 16 unique OTUs among the 22 ST2-positive samples (Table 2). Subtype 3 displayed the least intra-subtype diversity with only 12 unique OTUs among 101 ST3positive samples. Samples frequently contained multiple unique OTUs of ST1 and ST2, and up to three unique OTUs of ST1 or ST2 were detected in individual samples (Table 3). However, multiple OTUs of ST3 were not observed in the same sample. Furthermore, while unique OTUs of ST1 and ST2 were relatively evenly distributed among individual samples, two unique OTUs of ST3 were dominant in this population and were observed in 81 of 101 Blastocystis ST3-positive samples (Table 2).

\section{Association between sociodemographic variables and presence of Blastocystis}

Logistic regression analyses were performed to determine if any associations existed between Blastocystis infection and gender, adulthood, presence of symptoms, type of flooring, water source, sewage disposal, presence of domestic animals, presence of farm animals, presence 


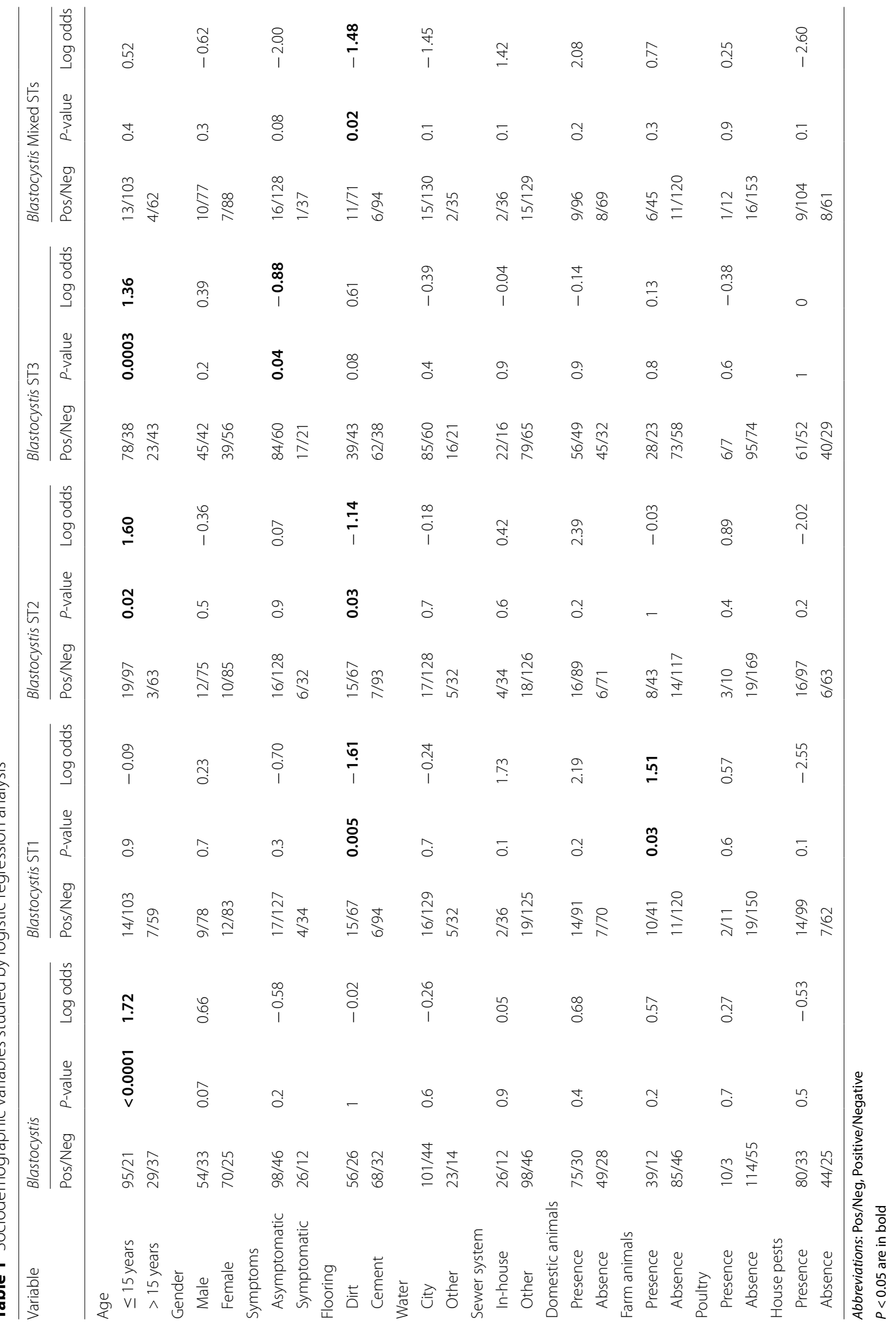



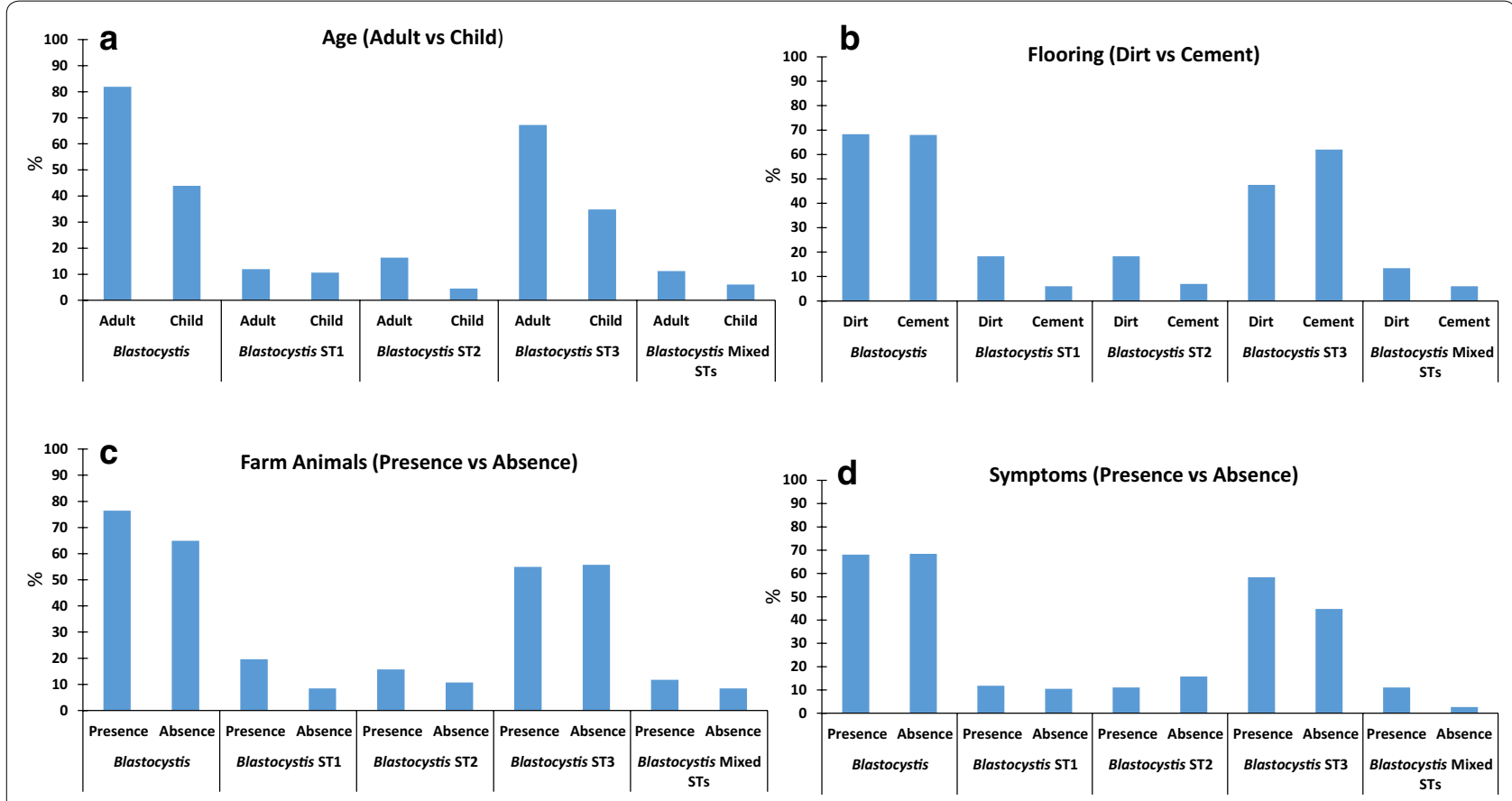

Fig. 1 Prevalence of Blastocystis (any subtype or subtype combination), ST1, ST2, ST3, and mixed STs considering the variables: (a) age (adult vs child); (b) type of flooring (dirt vs cement); (c) presence of farm animals (presence vs absence); and (d) presence of symptomatology (presence vs absence)

of chickens, or presence of house pests (Table 1). Adulthood was the only variable with a statistically significant association with Blastocystis infection (any subtype or subtype combination) (Fig. 1a). The odds of having Blastocystis was greater in the adult category (OR: 1.72, 95\% CI: 0.95-2.49, $P<0.0001$ ), and adulthood also had a statistically significant association with being ST2and ST3-positive when these outcomes were considered individually (OR: $1.6,95 \% \mathrm{CI}$ : $0.23-2.97, P=0.02$; OR: 1.36, 95\% CI: 0.62-2.09, $P=0.0003$ ) (Fig. 1a). Flooring type was also statistically significant for the outcomes of mixed STs, ST1- and ST2-positive (OR: -1.48 , 95\% CI: -2.70 to $-0.27, P=0.02$; OR: $-1.61,95 \% \mathrm{CI}:-2.74$ to $-0.48, P=0.005$; OR: $-1.14,95 \% \mathrm{CI}:-2.17$ to -0.11 , $P=0.03$ ) (Fig. 1b). The odds of having mixed STs, ST1 or ST2 infection were all found to be lower if a sample came from a person living in a home with a cement floor. This effect was not observed for Blastocystis infection (any subtype or subtype combination) or ST3 infections. The presence of farm animals increased the odds of being ST1-positive (OR: $1.51,95 \%$ CI: $0.18-2.84, P=0.03$ ) (Fig. 1c). Being symptomatic decreased the odds of being ST3 positive, although the association was weak (OR: -0.88 , 95\% CI: -1.73 to $-0.03, P=0.04$ ) (Fig. 1d). No other statistically significant associations between Blastocystis infection status or individual subtypes were found.

\section{Discussion}

Blastocystis is a common intestinal parasite found in humans and many other animals, and its prevalence and subtype distribution have been described in human populations from around the world [10]. However, molecular studies of Blastocystis in North America remain lacking. This study describes the prevalence and subtypes of Blastocystis present in a rural population in Mexico for which data on socioeconomic and demographic variables was also collected. This study also represents the first application of next-generation amplicon sequencing (NGS) of the Blastocystis SSU rRNA gene to a human population to characterize intra-host subtype diversity.

Fecal samples from 182 humans ranging in age from 2 to 51 years from a single rural community in Mexico were screened for the presence of Blastocystis by PCR of the SSU rRNA gene follow by NGS to determine subtypes. One or more Blastocystis subtype was found in $68.1 \%$ of the samples. While this prevalence is on par with other studies of Blastocystis prevalence in rural populations from both the Americas and other regions of the world, it is on the higher end of the reported prevalence of Blastocystis in humans. Diagnostic methods used in different studies could account for prevalence differences. Indeed, a lower prevalence was reported for Blastocystis for this same study population 
Table 2 Unique operational taxonomic units (OTUs) obtained for Blastocystis subtypes by next generation amplicon sequencing

\begin{tabular}{|c|c|c|c|}
\hline ST & $\begin{array}{l}\text { No. of unique OTUs } \\
\text { per subtype }\end{array}$ & $\begin{array}{l}\text { Unique OTU ID\# } \\
\text { (GenBank ID) }\end{array}$ & $\begin{array}{l}\text { No. of samples } \\
\text { containing } \\
\text { OTU }\end{array}$ \\
\hline \multirow[t]{15}{*}{ ST1 } & \multirow[t]{15}{*}{15} & 1a (MK874787) & 5 \\
\hline & & 1b (MK874795) & 4 \\
\hline & & 1c (MK874813) & 4 \\
\hline & & 1d (MK874786) & 3 \\
\hline & & 1e (MK874810) & 3 \\
\hline & & If (MK874789) & 2 \\
\hline & & 1g (MK874797) & 2 \\
\hline & & 1h (MK874807) & 2 \\
\hline & & 1i (MK874796) & 1 \\
\hline & & 1j (MK874798) & 1 \\
\hline & & 1k (MK874802) & 1 \\
\hline & & 1I (MK874816) & 1 \\
\hline & & $1 \mathrm{~m}(\mathrm{MK} 874817)$ & 1 \\
\hline & & 1n (MK874819) & 1 \\
\hline & & 10 (MK874822) & 1 \\
\hline \multirow[t]{16}{*}{ ST2 } & \multirow[t]{16}{*}{16} & 2a (MK874794) & 7 \\
\hline & & 2b (MK874785) & 6 \\
\hline & & 2c (MK874792) & 5 \\
\hline & & 2d (MK874793) & 4 \\
\hline & & 2e (MK874806) & 4 \\
\hline & & $2 f(M K 874790)$ & 3 \\
\hline & & 2g (MK874803) & 3 \\
\hline & & 2h (MK874814) & 2 \\
\hline & & 2i (MK874815) & 2 \\
\hline & & 2j (MK874804) & 1 \\
\hline & & 2k (MK874805) & 1 \\
\hline & & 2I (MK874808) & 1 \\
\hline & & 2m (MK874809) & 1 \\
\hline & & 2n (MK874811) & 1 \\
\hline & & 20 (MK874818) & 1 \\
\hline & & $2 p(M K 874821)$ & 1 \\
\hline \multirow[t]{12}{*}{ ST3 } & \multirow[t]{12}{*}{12} & 3а (MK874780) & 60 \\
\hline & & 3b (MK874781) & 21 \\
\hline & & 3c (MK874782) & 4 \\
\hline & & 3d (MK874783) & 4 \\
\hline & & 3e (MK874784) & 4 \\
\hline & & $3 f$ (MK874801) & 2 \\
\hline & & 3g (MK874788) & 1 \\
\hline & & 3h (MK874791) & 1 \\
\hline & & 3i (MK874799) & 1 \\
\hline & & 3j (MK874800) & 1 \\
\hline & & 3k (MK874812) & 1 \\
\hline & & 3I (MK874820) & 1 \\
\hline
\end{tabular}

using microscopy $(59.9 \% ; 109 / 182)$ with molecular confirmation in only $66 \%$ of those 109 microscopy positives [18].

From the 124 positive samples in this study, subtypes 1, 2 and 3 were found. These three subtypes are frequently reported in humans in the Americas and around the world [10]. Subtype 3 was the dominant subtype in this population, and was observed in $81.5 \%$ of Blastocystis-positive samples and $55.5 \%$ of the study population. Subtypes 1 and 2 were observed much less frequently in $16.9 \%$ and $17.7 \%$ of the positive samples, respectively. These results are similar to observations of Blastocystis subtypes from a multi-country study in South America and a study in Brazil where ST3 was the most prevalent subtype found among Blastocystis-positive human samples followed by ST1 and ST2 [8, 19]. Both studies reported other subtypes in low numbers, ST4-ST8, ST12, and novel subtypes in the multi-country study and ST4, ST6 and ST8 in the study from Brazil. However, no other Blastocystis subtypes were observed in the samples in this study. Similarly, in a study in the USA that examined 50 family units (101 adults and 38 children/adolescents) from Colorado only subtypes ST1 (20\%), ST2 (30\%), and ST3 (50\%) were identified [11]. In a survey of intestinal parasites in members of the Tapirapé ethnic group from the Brazilian Amazon region, only ST1, ST2 and ST3 were detected, but in their study ST1 was the most frequently identified subtype [20]. These differences between studies could be due to the geographical restrictions of some studies and may reflect population level or climatic influences on risk of infection with different Blastocystis subtypes.

The worldwide prevalence of Blastocystis mixed infections has been estimated to be $6 \%$ from previous studies on Blastocystis subtype diversity in humans [21]. This topic is not well explored, and only one study has directly addressed Blastocystis mixed subtype infections in humans [22]. By using a nested PCR assay that can identify subtypes 1 through 4, Scanlan et al. [22] demonstrated that $22 \%$ of Blastocystis-positive samples previously shown to contain a single subtype contained multiple Blastocystis subtypes. An advantage of NGS is its ability to assess intra-host subtype diversity [6]. The present study identified 17 mixed infections representing $13.7 \%$ of all Blastocystis infections in the population. Mixed subtype infections are underrepresented compared to expectations from subtype prevalence. However, mixed infections in our study were within the range of observations from other human studies [21, 
Table 3 Blastocystis prevalence for each subtype in mono-infections and for the different subtype combinations in mixed subtype infections

\begin{tabular}{|c|c|c|c|c|c|c|c|c|}
\hline & \multicolumn{4}{|c|}{ Mono subtype infections } & \multicolumn{4}{|c|}{ Mixed subtype infections } \\
\hline & ST1 only & ST2 only & ST3 only & $\begin{array}{l}\text { Total mono- } \\
\text { infections }\end{array}$ & ST1/ST3 mix & ST2/ST3 mix & ST1/ST2/ST3 mix & $\begin{array}{l}\text { Total } \\
\text { mixed } \\
\text { infections }\end{array}$ \\
\hline Total positive samples & 9 & 14 & 84 & 107 & 9 & 5 & 3 & 17 \\
\hline Percentage of all samples & 4.9 & 7.7 & 46.2 & 58.8 & 4.9 & 2.7 & 1.6 & 9.2 \\
\hline Percentage of positive samples & 7.3 & 11.3 & 67.7 & 86.3 & 7.3 & 4.0 & 2.4 & 13.7 \\
\hline Percentage of mixed samples & na & na & na & na & 52.9 & 29.4 & 17.6 & na \\
\hline
\end{tabular}

Abbreviations: na, not applicable

22]. Combinations of ST1 + ST3, ST2 + ST3, and ST1 $+\mathrm{ST} 2+\mathrm{ST} 3$ were all observed. ST3 was observed in all mixed Blastocystis infections, ST1 in 12 mixed infections, and ST2 in eight mixed infections. Although the population studied here was limited in its Blastocystis subtype diversity, these data support the use of NGS for exploring mixed subtype infections in humans.

Intra-subtype variability could play a role in understanding Blastocystis transmission and pathogenicity $[6,14,23-25]$. In the present study, intra-subtype variability varied widely between the subtypes. While ST1 and ST2 had high proportions of unique sequences, ST3 was surprisingly homogeneous. Unique sequences represented $71.4 \%$ of ST1 sequences and $72.7 \%$ of ST2 sequences, but only $11.9 \%$ of ST3 sequences were unique. Two OTUs of ST3 also dominated in this population representing $80.2 \%$ of all ST3 observations. This lower level of intra-subtype variability has been reported before for ST3 both in humans and cattle [6, 26]. There was also no within-host variation in ST3 despite multiple OTUs of ST1 or ST2 being frequently observed in the same host. The homogeneity of ST3 in this population may indicate that ST3 is highly endemic in this community and may be acquired from a common source or may pass more easily between humans than the other subtypes observed in this study.

In previous studies Blastocystis infection has been associated with factors such as age, animal contact, and sanitation practices [27-29]. To better understand what factors might influence Blastocystis infection risk in this population, demographic and socioeconomic data was collected and logistic regression analysis was used to determine if any associations exist between having Blastocystis, mixed STs, ST1, ST2 or ST3 infections and any of these factors. Because only two of the 43 unique OTUs detected in this study were found in more than 10 samples, statistical analyses attempting to link unique OTUs with risk factors were not performed.
In this population, the only factor associated with having Blastocystis was adulthood, with the odds of being Blastocystis-positive being greater in the adult ( $>15$ years-old) category (OR: $1.72, P \leq 0.0001)$. In the present study, 116 of the 182 samples were adults ( $>15$ yearsold) and $81.9 \%$ were Blastocystis-positive while $43.9 \%$ of the 66 children were Blastocystis-positive. Age has been indicated as a risk factor for Blastocystis in other studies, and human infants and young animals tend to have lower infection rates than adults [6, 29-31]. Adulthood was also associated with increased odds of having an ST2 or an ST3 infection (OR: $1.6, P=0.02$; OR: $1.36, P=0.03$ ), but this association was not observed for mixed or ST1 infections. These results could indicate that behavioral or physiological differences between adults and children may be important in determining infection risk for specific subtypes of Blastocystis.

Flooring material was significantly associated with mixed, ST1, and ST2 infections, and the odds of having one of these types of infections was decreased in homes with cement floors (OR: $-1.48, P=0.02$; OR: $-1.61, P=$ 0.005 ; OR: $-1.14, P=0.03)$. This outcome may capture some socioeconomic effect such as improved hygiene not directly measured in this study as socioeconomic status has been associated with infection risk previously [30]. Having farm animals significantly increased the odds of having ST1 (OR: $1.51, P=0.03$ ). No other subtypes were associated with animals in this study. These results may indicate that zoonotic transmission of ST1 occurs in this population although data on the subtypes circulating in the animals owned or handled by ST1-positive individuals would be necessary to confirm this finding. Notably, artiodactyls such as pigs and cattle are the second most common host reported for ST1 after humans, further supporting the potential for zoonotic transmission of this subtype [10].

Being symptomatic, defined as answering yes to one or more Rome III criteria, was significantly associated 
with ST3 infection, although the association was negative (OR: $-0.88, P=0.04)$. Being symptomatic decreased the odds of having ST3. This association while significant was not strong and caution should be taken in overinterpretation of this result as associations between subtypes and symptomatology is still unclear. Some studies have indicated that there is no association between diarrhea and ST1, ST2 or ST3, but ST4 is associated with diarrhea and irritable bowel syndrome [32-34]. However, ST1 and ST3 have been associated with intestinal symptoms in other studies $[35,36]$.

Subtype 1 has been found in drinking water in Thailand and river water in Nepal, and waterborne transmission of Blastocystis to humans was suspected in these studies [28]. In the present study, no statistically significant association was found between infection and water source indicating that transmission in this community may occur through other routes. No statistically significant associations were found between any infection outcomes and sewage disposal, presence of domestic animals, presence of chickens, or presence of house pests.

\section{Conclusions}

This study provides important information about the epidemiology of Blastocystis and represents the first application of a Blastocystis-specific NGS protocol to study Blastocystis in humans. Although the study population described had a relatively homogenous Blastocystis subtype community, infection status and individual subtypes could still be linked to specific risk factors. More studies which aim to characterize mixed subtype infections and intra-subtype variation are needed to understand the transmission dynamics, epidemiology, and pathogenicity of Blastocystis in humans and animals. NGS provides a valuable tool for achieving this goal.

\section{Supplementary information}

Supplementary information accompanies this paper at https://doi. org/10.1186/s13071-019-3814-z.

Additional file 1: Table S1. Blastocystis subtypes relative abundance in positive samples identified by next generation amplicon sequencing.

\section{Abbreviations}

PCR: polymerase chain reaction; SSU rRNA: small subunit of the ribosomal RNA; ST: subtype; OTU: operational taxonomic unit.

\footnotetext{
Acknowledgments

The authors thank Nadja George, Tobías Portillo, Eric G. Hernández, Miriam E. Nieves-Ramírez, Oswaldo Partida-Rodríguez and Ulises Magaña for their technical support of this study. We also thank Dr Luis E. Equiarte and Dr Daniel Piñero for their constructive comments to the manuscript. The authors are grateful to the Health Ministry of the state of Morelos, Mexico for supporting this study in the community of Xoxocotla. LR-V is a doctoral student from Programa de Doctorado en Ciencias Biomédicas, Universidad Nacional Autónoma
}

de México (UNAM) and was supported by the fellowship 348424/239901 from CONACYT. This paper constitutes a partial fulfilment of the Graduate Program.

\section{Authors' contributions}

LRV, JM, MS and CX contributed to the conception and design of the experiments. CX, LRV, PM and ASV managed the field collections and acquisition of sociodemographic data. LRV, EG and HPJ performed sample processing and DNA extractions. JM conducted the next-generation sequencing experiments. $\mathrm{JM}, \mathrm{MS}$ and $\mathrm{AM}$ analyzed next-generation sequencing data and conducted bioinformatic and statistical analysis. JM and MS wrote the manuscript. All authors contributed to the revision of the manuscript. All authors read and approved the final manuscript.

\section{Funding}

The present work was supported by Grants IN215018, N202408 and IN218214 from PAPIIT (DGAPA), Universidad Nacional Autónoma de México (UNAM), 2016-1 272601, 00257091 and 0362010 from the National Council for Science and Technology in Mexico (CONACYT). Liliana Rojas-Velázquez was supported by the fellowship 348424/239901 from CONACYT.

\section{Availability of data and materials}

All raw fasta files were deposited to the NCBI sequence read archive under the accession number PRJNA523857. The nucleotide sequences for unique OTUs obtained in this study have been deposited in the GenBank database under the accession numbers MK874780-MK874822.

\section{Ethics approval and consent to participate}

Sample collections in this study were conducted under the ethical principles and approval of both the Mexican Commission on Ethics and Research of the Health Ministry of the state of Morelos (Comisiones de Ética y de Investigación del Ministerio de Salud del Estado de Morelos); and the Commission on Ethics in Research of the Facultad de Medicina of the Universidad Nacional Autónoma de México (UNAM) (Comité de Ética de Investigación de la Facultad de Medicina de la Universidad Nacional Autónoma de México). The guidelines of the committees are based on the Mexican Official Norm (Norma Oficial Mexicana NOM-012-SSA3-2007), which regulates the ethical principles of every research on humans and on laboratory animals, as well as on the Declaration of Helsinki, which set ethical principles regarding human experimentation developed by the World Health Organization (WHO). Based on these guidelines, our study only used samples from volunteers, who were informed about research objectives and sampling procedures. An informed consent letter was obtained from all participants or from one parent of each minor (under 18 years-old).

\section{Consent for publication}

Not applicable.

\section{Competing interests}

The authors declare that they have no competing interests.

\section{Author details}

${ }^{1}$ Unidad de Investigación en Medicina Experimental, Facultad de Medicina, Universidad Nacional Autónoma de México (UNAM), Mexico City, Mexico. ${ }^{2}$ Environmental Microbial and Food Safety Laboratory, Agricultural Research Service, United States Department of Agriculture, Beltsville, MD, USA. ${ }^{3}$ Unidad de Posgrado, Universidad Nacional Autónoma de México (UNAM), Mexico City, Mexico.

Received: 10 September 2019 Accepted: 19 November 2019

Published online: 27 November 2019

\section{References}

1. Andersen LO, Stensvold CR. Blastocystis in Health and Disease: Are we moving from a clinical to a public health perspective? J Clin Microbiol. 2016;54:524-8.

2. Ajjampur SSR, Tan KSW. Pathogenic mechanisms in Blastocystis spp. Interpreting results from in vitro and in vivo studies. Parasitol Int. 2016;65:772-9. 
3. Casero RD, Mongi F, Sánchez A, Ramírez JD. Blastocystis and urticaria: examination of subtypes and morphotypes in an unusual clinical manifestation. Acta Trop. 2015;148:156-61.

4. Tan KSW. New insights on classification, identification, and clinical relevance of Blastocystis spp. Clin Microbiol Rev. 2008;21:639-65.

5. Alfellani MA, Taner-Mulla D, Jacob AS, Imeede CA, Yoshikawa H, Stensvold CR, et al. Genetic diversity of Blastocystis in livestock and zoo animals. Protist. 2013;164:497-509.

6. Maloney JG, Lombard JE, Shivley CB, Urie NJ, Santin M. Zoonotic and genetically diverse subtypes of Blastocystis in US pre-weaned dairy heifer calves. Parasitol Res. 2019;118:575-82.

7. Zhao GH, Hu XF, Liu TL, Hu RS, Yu ZQ, Yang WB, et al. Molecular characterization of Blastocystis sp. in captive wild animals in Qinling Mountains. Parasitol Res. 2017:116:2327-33.

8. Ramírez JD, Sánchez A, Hernández C, Flórez C, Bernal MC, Giraldo JC, et al. Geographic distribution of human Blastocystis subtypes in South America. Infect Genet Evol. 2016;41:32-5.

9. Stensvold CR, Clark CG. Current status of Blastocystis: a personal view. Parasitol Int. 2016;65:763-71.

10. Stensvold CR. Blastocystis. Biology of foodborne parasites. Boca Raton: Taylor and Francis Group; 2015. p. 53-75.

11. Scanlan PD, Knight R, Song SJ, Ackermann G, Cotter PD. Prevalence and genetic diversity of Blastocystis in family units living in the United States. Infect Genet Evol. 2016;45:95-7.

12. Vargas-Sanchez G-B, Romero-Valdovinos M, Ramirez-Guerrero C, VargasHernandez I, Ramirez-Miranda ME, Martinez-Ocaña J, et al. Blastocystis isolates from patients with irritable bowel syndrome and from asymptomatic carriers exhibit similar parasitological loads, but significantly different generation times and genetic variability across multiple subtypes. PLoS One. 2015;10:e0124006.

13. Jimenez-Gonzalez DE, Martinez-Flores WA, Reyes-Gordillo J, RamirezMiranda ME, Arroyo-Escalante S, Romero-Valdovinos M, et al. Blastocystis infection is associated with irritable bowel syndrome in a Mexican patient population. Parasitol Res. 2012;110:1269-75.

14. Maloney JG, Molokin A, Santin M. Next generation amplicon sequencing improves detection of Blastocystis mixed subtype infections. Infect Genet Evol. 2019;73:119-25.

15. Santín M, Gómez-Muñoz MT, Solano-Aguilar G, Fayer R. Development of a new PCR protocol to detect and subtype Blastocystis spp. from humans and animals. Parasitol Res. 2011;109:205-12.

16. Bushnell, B. (2017). BBTools software package. http://sourceforge.net/ projects/bbmap. Accessed 6 June 2019

17. Rognes T, Flouri T, Nichols B, Quince C, Mahé F. VSEARCH: a versatile open source tool for metagenomics. PeerJ. 2016;4:e2584

18. Rojas-Velázquez L, Morán P, Serrano-Vázquez A, Fernández LD, PérezJuárez H, Poot-Hernández AC, et al. Genetic diversity and distribution of Blastocystis subtype 3 in human populations, with special reference to a rural population in central Mexico. BioMed Res Int. 2018;2018:3916263.

19. Seguí R, Muñoz-Antoli $C$, Klisiowicz DR, Oishi CY, Köster PC, De Lucio A, et al. Prevalence of intestinal parasites, with emphasis on the molecular epidemiology of Giardia duodenalis and Blastocystis sp., in the Paranaguá Bay, Brazil: a community survey. Parasit Vectors. 2018;11:490.

20. Malheiros AF, Stensvold CR, Clark CG, Braga GB, Shaw JJ. Short report: Molecular characterization of Blastocystis obtained from members of the indigenous tapirapé ethnic group from the Brazilian Amazon Region, Brazil. Am J Trop Med Hyg. 2011;85:1050-3.

21. Alfellani MA, Stensvold CR, Vidal-Lapiedra A, Onuoha ESU, Fagbenro-Beyioku AF, Clark CG. Variable geographic distribution of Blastocystis subtypes and its potential implications. Acta Trop. 2013;126:11-8.
22. Scanlan PD, Stensvold CR, Cotter PD. Development and application of a Blastocystis subtype-specific PCR assay reveals that mixed-subtype infections are common in a healthy human population. Appl Environ Microbiol. 2015;81:4071-6.

23. Clark CG. Extensive genetic diversity in Blastocystis hominis. Mol Biochem Parasitol. 1997:87:79-83.

24. Fayer $R$, Santin M, Macarisin D. Detection of concurrent infection of dairy cattle with Blastocystis, Cryptosporidium, Giardia, and Enterocytozoon by molecular and microscopic methods. Parasitol Res. 2012:111:1349-55.

25. Fayer R, Elsasser T, Gould R, Solano G, Urban J, Santin M. Blastocystis tropism in the pig intestine. Parasitol Res. 2014;113:1465-72.

26. Beghini F, Pasolli E, Truong TD, Putignani L, Cacciò SM, Segata N. Largescale comparative metagenomics of Blastocystis, a common member of the human gut microbiome. ISME J. 2017;11:2848-63.

27. Lee IL, Tan TC, Tan PC, Nanthiney DR, Biraj MK, Surendra KM, et al. Predominance of Blastocystis sp. subtype 4 in rural communities, Nepal. Parasitol Res. 2012;110:1553-62.

28. Leelayoova S, Siripattanapipong S, Thathaisong U, Naaglor T, Taamasri P, Piyaraj P, et al. Drinking water: a possible source of Blastocystis spp. subtype 1 infection in schoolchildren of a rural community in central Thailand. Am J Trop Med Hyg. 2008;79:401-6.

29. Pandey PK, Verma P, Marathe N, Shetty S, Bavdekar A, Patole MS, et al. Prevalence and subtype analysis of Blastocystis in healthy Indian individuals. Infect Genet Evol. 2015;31:296-9.

30. Abdulsalam AM, Ithoi I, Al-Mekhlafi HM, Khan AH, Ahmed A, Surin J, et al. Prevalence, predictors and clinical significance of Blastocystis sp. in Sebha, Libya. Parasit Vectors. 2013;6:86.

31. Mohammad NA, Al-Mekhlafi HM, Moktar N, Anuar TS. Prevalence and risk factors of Blastocystis infection among underprivileged communities in rural Malaysia. Asian Pac J Trop Med. 2017;10:491-7.

32. Domínguez-Márquez MV, Guna R, Muñoz C, Gómez-Muñoz MT, Borrás R. High prevalence of subtype 4 among isolates of Blastocystis hominis from symptomatic patients of a health district of Valencia (Spain). Parasitol Res. 2009;105:949-55.

33. Mattiucci S, Crisafi B, Gabrielli S, Paoletti M, Cancrini G. Molecular epidemiology and genetic diversity of Blastocystis infection in humans in Italy. Epidemiol Infect. 2016;144:635-46.

34. Stensvold CR, Christiansen DB, Elisabeth K, Olsen P, Nielsen HV. Short report: Blastocystis sp. subtype 4 is common in Danish Blastocystispositive patients presenting with acute diarrhea. Am J Trop Med Hyg. 2011;84:883-5

35. Jones MS, Whipps CM, Ganac RD, Hudson NR, Boroom K. Association of Blastocystis subtype 3 and 1 with patients from an Oregon community presenting with chronic gastrointestinal illness. Parasitol Res. 2009;104:341-5.

36. Moosavi A, Haghighi A, Mojarad EN, Zayeri F, Alebouyeh M, Khazan H, et al. Genetic variability of Blastocystis sp. Isolated from symptomatic and asymptomatic individuals in Iran. Parasitol Res. 2012;111:2311-5.

\section{Publisher's Note}

Springer Nature remains neutral with regard to jurisdictional claims in published maps and institutional affiliations.

Ready to submit your research? Choose BMC and benefit from:

- fast, convenient online submission

- thorough peer review by experienced researchers in your field

- rapid publication on acceptance

- support for research data, including large and complex data types

- gold Open Access which fosters wider collaboration and increased citations

- maximum visibility for your research: over 100M website views per year

At $\mathrm{BMC}$, research is always in progress.

Learn more biomedcentral.com/submissions 